\title{
Papers
}

\section{Increase in blood glucose concentration during antihypertensive treatment as a predictor of myocardial infarction: population based cohort study}

\author{
Kristina Dunder, Lars Lind, Björn Zethelius, Lars Berglund, Hans Lithell
}

\begin{abstract}
Objective To investigate the impact of an increase in blood glucose on the risk of developing myocardial infarction, with particular emphasis on people taking antihypertensive drugs.

Design Prospective population based cohort study.

Setting Uppsala, Sweden.

Participants 1860 men who had participated in

1970-3 at age 50 in a health survey aimed at identifying risk factors for cardiovascular disease and were re-examined at age 60 and then followed for 17.4 years.
\end{abstract}

Main outcome measure Myocardial infarction after age 60 .

Results The incidence of myocardial infarction was significantly higher in men treated for hypertension than in those without such treatment (23\% v 13.5\%, $\mathrm{P}<0.0001)$. Participants who developed myocardial infarction after the age of $60(n=253)$ showed a significantly larger increase in blood glucose between age 50 and 60 than did those without myocardial infarction. In multivariate Cox proportional hazard models increase in blood glucose was an independent risk factor for myocardial infarction $(\mathrm{P}=0.0001)$ in men receiving antihypertensive treatment at age 60 ( $\mathrm{n}=291$, mainly $\beta$ blockers and thiazide diuretics) but not in those without such treatment. The impact of increase in blood glucose declined after inclusion of serum proinsulin concentrations at baseline but was still significant. A significant interaction existed between proinsulin concentration (a marker of insulin resistance) at baseline and antihypertensive treatment on increase in blood glucose.

Conclusions Increase in blood glucose between the ages of 50 and 60 and baseline proinsulin concentration were important risk factors for myocardial infarction in men receiving antihypertensive treatment, indicating that both an insulin resistant state and the metabolic impact of $\beta$ blockers and diuretics increase the risk of myocardial infarction.

\section{Introduction}

During the past decade several studies have shown that a large proportion of patients with hypertension are resistant to insulin stimulated glucose uptake and are hyperinsulinaemic compared with normotensive controls. $^{1-4}$ Treatment with $\beta$ blockers or thiazide diuretics further increases insulin resistance, ${ }^{56}$ thereby increasing the risk of developing type 2 diabetes mellitus or impaired glucose tolerance..$^{7-11}$

Diabetes mellitus and impaired glucose tolerance are both associated with an increased risk of coronary heart disease, ${ }^{12}{ }^{13}$ but whether these conditions when induced by $\beta$ blockers or thiazides are associated with increased risk of coronary heart disease is unknown. A review of trials in which diuretics were used as antihypertensive treatment concluded that a definite association existed between diuretic treatment and development of impaired glucose tolerance but that this development did not increase the risk of coronary heart disease. ${ }^{14}$ Furthermore, Samuelsson et al found that drug related development of diabetes mellitus did not increase the risk of coronary events over a 15 year treatment period in 686 hypertensive men. ${ }^{15}$

We investigated the impact of variations in fasting blood glucose, blood pressure, and body mass index between the ages of 50 and 60 on the risk of developing a myocardial infarction after this 10 year period (mean follow up 17.4 years) in a population based sample of men. In the analysis we paid special attention to the men receiving antihypertensive treatment, with the hypothesis that a drug induced increase in fasting blood glucose would increase the risk of myocardial infarction.

\section{Methods}

The Uppsala longitudinal study of men is a population based study aimed at identifying risk factors for cardiovascular disease. Between 1970 and 1973 all men born in 1920-4 and resident in the municipality of Uppsala, Sweden, were invited to participate in a health survey, and 2322 (82\%) of the 2841 invited men participated. All participants gave informed consent. These investigations have been extensively described. ${ }^{16}$

At the age of 50 the men underwent a physical examination, including measurement of height, weight, and radial pulse rate. The investigators measured office blood pressure twice to the nearest $5 \mathrm{~mm} \mathrm{Hg}$, in the supine position after 10 minutes' rest, with a mercury sphygmomanometer and recorded the mean value.
Department of Public Health and Caring Sciences/ Geriatrics, Uppsala University, $\mathrm{PO}$ Box 609, SE-751 25 Uppsala, Sweden Kristina Dunder medical doctor Björn Zethelius medical doctor Hans Lithell professor Lars Berglund statistician

Department of Medical Sciences, Uppsala University, Akademiska sjukhuset, SE-751 85 Uppsala, Sweden Lars Lind professor

Correspondence to: K Dunder kristina.dunder@ pubcare.uu.se

bmj.com 2003;326:681 
They drew blood samples in the morning after an overnight fast and analysed blood glucose and serum insulin. In 1980 total cholesterol, high density lipoprotein cholesterol, and triglycerides were analysed in serum that had been frozen since the baseline examinations. The concentration of low density lipoprotein cholesterol was calculated by using Friedewald's formula. The methods have been described elsewhere. ${ }^{8}$ In 1995-8 researchers in Cambridge, United Kingdom, analysed the concentrations of intact and 32-33 split proinsulin, by using the two site immunometric assay technique. ${ }^{17}$ These analyses were made in all available serum samples $(\mathrm{n}=1335)$.

Between 1980 and 1984 the investigators re-examined eligible men (at age 60) who had participated in the first survey-1860/2130 (87.5\%) men participated. This examination included anthropometric data, blood pressure, and blood samples. The investigators collected data on admissions to hospital or death from myocardial infarction (ICD-9 code 410) or angina pectoris (ICD 413) from the official cause of death and hospital registers.

We based our study on the 1860 men who participated in both the baseline investigations at age 50 and the re-examination at age 60 . We excluded participants who had been admitted to hospital for myocardial infarction before the examination at age 60 , as well as those without myocardial infarction but with angina pectoris (according to in-hospital registers) during the follow up period.

We grouped the population into participants receiving and not receiving antihypertensive treatment at age 60. Three hundred and sixteen men had received antihypertensive treatment, of whom 82 had a non-selective $\beta$ blocker, 65 had a selective $\beta$ blocker, 66 had a thiazide diuretic, and 103 received a combination of $\beta$ blocker and thiazide diuretic. Forty one participants had hydralazine added to the treatment. After exclusion of participants with myocardial infarction or angina pectoris before the age of 60, 291 men in the group with antihypertensive treatment and 1358 men in the group without such treatment remained at age 60 .

\section{Statistical analysis}

We evaluated differences in metabolic characteristics between groups by factorial analysis of variance, and we used stepwise multiple Cox proportional hazard analysis (backward selection) to evaluate the independent power of the risk factors. We used Z transformed standardised variables in the Cox proportional hazard analysis to make hazard ratios comparable. To make hazard ratios comparable between the groups we used a common standard deviation for the two groups for each variable in standardising the variables.

We log transformed non-normally distributed variables (change in glucose, blood glucose, serum insulin, proinsulin, split proinsulin, and serum triglycerides) tested by Shapiro-Wilk's test $(\mathrm{w}<0.95)$ before we analysed them. We calculated the risk associated with a $10 \%$ increase in blood glucose as follows: hazard ratio** $(\log (1.1) / \mathrm{SD})$; for example, $1.37 * *(\log (1.1)$ $0.16)=1.37 * * 0.596=1.206$. We regarded $\mathrm{P}<0.05$ as significant.

\section{Results}

Table 1 presents means and standard deviations for metabolic characteristics at age 50 and for changes in fasting blood glucose, blood pressure, and body mass index between age 50 and 60 , as well as the effect of these variables on the risk of having a myocardial infarction after age 60 .

Participants being treated for hypertension at age 60 showed higher concentrations of indices of the metabolic syndrome, such as serum proinsulin, fasting blood glucose, and serum triglycerides, as well as a larger increase in fasting blood glucose between the ages of 50 and 60 compared with those without such treatment. The mean blood pressure in the treatment group was 153/96 $\mathrm{mm} \mathrm{Hg}$ at baseline (age 50) and $154 / 93 \mathrm{~mm} \mathrm{Hg}$ at age 60.

Table 1 Metabolic characteristics at age 50, changes between age 50 and 60, and effect of these variables on risk of having a myocardial infarction after age 60

\begin{tabular}{|c|c|c|c|c|c|}
\hline \multirow[b]{2}{*}{ Characteristic } & \multicolumn{2}{|c|}{ Antihypertensive treatment $(\mathrm{n}=291)$} & \multicolumn{2}{|c|}{ No antihypertensive treatment $(\mathrm{n}=\mathbf{1 3 5 8})$} & \multirow[b]{2}{*}{$P$ value } \\
\hline & Mean (SD) & Hazard ratio* $(95 \% \mathrm{Cl})$ & Mean (SD) & Hazard ratio* $(95 \% \mathrm{Cl})$ & \\
\hline \multicolumn{6}{|l|}{ Age 50 (baseline) } \\
\hline Blood glucose (mmol/l) & $5.09(0.72)$ & $1.06(0.83$ to 1.30$)$ & $4.96(0.63)$ & $1.12(0.98$ to 1.26$)$ & 0.008 \\
\hline Body mass index $\left(\mathrm{kg} / \mathrm{m}^{2}\right)$ & $26.0(3.41)$ & $1.16(0.93$ to 1.44$)$ & $24.6(2.99)$ & 1.20 (1.03 to 1.38$)$ & $<0.0001$ \\
\hline Immunoreactive insulin $(\mu \mathrm{U} / \mathrm{ml})$ & $14.4(7.71)$ & $1.05(0.80$ to 1.38$)$ & $12.4(7.30)$ & $1.07(0.91$ to 1.26$)$ & $<0.0001$ \\
\hline Intact proinsulin (pmol/l) & $3.69(3.92)$ & 1.59 (1.20 to 2.10$)$ & $2.78(2.69)$ & 1.54 (1.28 to 1.84$)$ & 0.0004 \\
\hline Split proinsulin (pmol/l) & $8.98(9.08)$ & 1.48 (1.12 to 1.94$)$ & $6.59(5.84)$ & 1.26 (1.04 to 1.52$)$ & $<0.0001$ \\
\hline LDL cholesterol (mmol/l) & $5.39(1.29)$ & 1.36 (1.08 to 1.66$)$ & $5.23(1.24)$ & 1.35 (1.18 to 1.53$)$ & 0.08 \\
\hline HDL cholesterol (mmol/l) & $1.32(0.37)$ & 0.77 (0.55 to 1.06$)$ & $1.38(0.38)$ & 0.76 (0.63 to 0.91$)$ & 0.04 \\
\hline LDL:HDL cholesterol ratio & $4.44(1.96)$ & 1.29 (1.09 to 1.48$)$ & $4.12(1.59)$ & 1.44 (1.25 to 1.65$)$ & 0.008 \\
\hline Serum triglycerides $(\mathrm{mmol} / \mathrm{l})$ & $2.10(1.15)$ & $1.16(0.88$ to 1.35$)$ & $1.84(1.12)$ & 1.29 (1.12 to 1.48$)$ & $<0.0001$ \\
\hline Systolic blood pressure $(\mathrm{mm} \mathrm{Hg})$ & $153(20.9)$ & $1.02(0.83$ to 1.25$)$ & $128(13.6)$ & 1.22 (1.02 to 1.45$)$ & $<0.0001$ \\
\hline Diastolic blood pressure $(\mathrm{mm} \mathrm{Hg})$ & $96(11.7)$ & 1.07 (0.85 to 1.33$)$ & $80(8.50)$ & 1.26 (1.04 to 1.51$)$ & $<0.0001$ \\
\hline \multicolumn{6}{|l|}{ Change from age 50 to 60} \\
\hline Glucose & $0.38(1.51)$ & $1.38(1.16$ to 1.60$)$ & $-0.06(0.94)$ & $1.08(0.92$ to 1.26$)$ & $<0.0001$ \\
\hline Body mass index & $0.66(1.96)$ & 0.85 (0.68 to 1.08$)$ & $0.46(1.71)$ & 0.94 (0.81 to 1.10$)$ & 0.07 \\
\hline Systolic blood pressure & $0.5(24.2)$ & 0.96 (0.80 to 1.16$)$ & $12(15.5)$ & 1.22 (1.04 to 1.43 ) & $<0.0001$ \\
\hline Diastolic blood pressure & $-3(12.4)$ & 0.90 (0.74 to 1.10$)$ & $6(8.70)$ & 0.92 (0.78 to 1.09 ) & $<0.0001$ \\
\hline
\end{tabular}

LDL=low density lipoprotein; HDL=high density lipoprotein.

*For risk of having myocardial infarction after age 60 . Hazard ratios for a one standard deviation variation in the variables.

†P value for differences between means. 
Table 2 Effects of changes between age 50 and 60 on the risk of having a myocardial infarction after age 60 , after adjustment for effect of corresponding baseline values at age 50

\begin{tabular}{|c|c|c|c|c|}
\hline \multirow[b]{2}{*}{ Risk factor } & \multicolumn{2}{|c|}{ Antihypertensive treatment $(\mathrm{n}=291)$} & \multicolumn{2}{|c|}{ No antihypertensive treatment ( $n=1358$ ) } \\
\hline & Hazard ratio* $(95 \% \mathrm{CI})$ & $P$ value & Hazard ratio* $(95 \% \mathrm{Cl})$ & $P$ value \\
\hline Change in glucose & 1.37 (1.16 to 1.59$)$ & 0.0004 & 1.14 (0.98 to 1.32$)$ & 0.10 \\
\hline Blood glucose (50) & 1.04 (0.83 to 1.28$)$ & 0.72 & 1.16 (1.01 to 1.31$)$ & 0.03 \\
\hline Change in body mass index & 0.88 (0.69 to 1.11$)$ & 0.29 & $0.98(0.84$ to 1.14$)$ & 0.78 \\
\hline Body mass index (50) & $1.11(0.88$ to 1.46$)$ & 0.36 & 1.19 (1.02 to 1.38$)$ & 0.02 \\
\hline Change in systolic blood pressure & 0.96 (0.75 to 1.22$)$ & 0.74 & 1.25 (1.07 to 1.46$)$ & 0.006 \\
\hline Systolic blood pressure (50) & 0.99 (0.75 to 1.30$)$ & 0.98 & $1.27(1.06$ to 1.50$)$ & 0.01 \\
\hline Change in diastolic blood pressure & 0.85 (0.61 to 1.17$)$ & 0.32 & 1.01 (0.84 to 1.21$)$ & 0.92 \\
\hline Diastolic blood pressure (50) & 0.92 (0.63 to 1.34$)$ & 0.67 & 1.26 (1.03 to 1.54$)$ & 0.02 \\
\hline
\end{tabular}

*Hazard ratios for a one standard deviation (given in table 1) variation in the variables.

Table 3 Stepwise multivariate Cox proportional hazard analysis of variables identified in univariate analysis as risk factors for myocardial infarction after age 60

\begin{tabular}{|c|c|c|c|c|}
\hline \multirow[b]{2}{*}{ Risk factor } & \multicolumn{2}{|c|}{ Excluding serum proinsulin } & \multicolumn{2}{|c|}{ Including serum proinsulin } \\
\hline & Hazard ratio $(95 \% \mathrm{Cl})$ & $P$ value & Hazard ratio $(95 \% \mathrm{Cl})$ & $P$ value \\
\hline \multicolumn{5}{|l|}{ Antihypertensive treatment $(\mathrm{n}=291)$} \\
\hline Change in glucose & $1.50(1.25$ to 1.78$)$ & 0.0001 & 1.29 (1.06 to 1.55$)$ & 0.01 \\
\hline LDL:HDL cholesterol ratio & 1.32 (1.10 to 1.51$)$ & 0.004 & $1.28(1.05$ to 1.49$)$ & 0.02 \\
\hline Intact proinsulin & NA & NA & 1.33 (1.01 to 1.78$)$ & 0.05 \\
\hline \multicolumn{5}{|c|}{ No antihypertensive treatment $(\mathrm{n}=\mathbf{1 3 5 8})$} \\
\hline Change in glucose & $1.04(0.86$ to 1.24$)$ & 0.72 & $0.99(0.81$ to 1.21$)$ & 0.98 \\
\hline LDL:HDL cholesterol ratio & $1.42(1.22$ to 1.67$)$ & $<0.0001$ & 1.39 (1.18 to 1.61$)$ & 0.0001 \\
\hline Change in systolic blood pressure & 1.24 (1.03 to 1.48$)$ & 0.02 & $1.26(1.01$ to 1.55$)$ & 0.04 \\
\hline Intact proinsulin & NA & NA & 1.38 (1.13 to 1.67$)$ & 0.0016 \\
\hline
\end{tabular}

LDL=low density lipoprotein; $H D L=$ high density lipoprotein; NA=not applicable.

*Hazard ratios for a one standard deviation (given in table 1) variation in the variables.

Participants who developed myocardial infarction after the age of 60 ( $n=253$ over the 17.4 year follow up) showed a significantly larger increase in fasting blood glucose than those who did not develop myocardial infarction (0.28 (SD 1.61) mmol/l $v-0.04(0.94)$ $\mathrm{mmol} / \mathrm{l}, \mathrm{P}=0.001)$. The incidence of myocardial infarction was significantly higher in men treated for hypertension than in those without treatment $-67 / 291$ $(23.0 \%) v 183 / 1358(13.5 \%), \mathrm{P}<0.0001$.

Table 2 shows hazard ratios for a one standard deviation variation in fasting blood glucose, body mass index, and systolic and diastolic blood pressure between age 50 and age 60 , after adjustment for baseline values of these factors. An increase in fasting blood glucose predicted myocardial infarction in the group receiving antihypertensive treatment but not in the group without such treatment. An increase of 10\% in fasting blood glucose in the treatment group was associated with a $21.7 \%$ higher risk of myocardial infarction after age 60 (data not shown). However, an increase in systolic blood pressure was a significant predictor of future myocardial infarction only in participants without antihypertensive treatment.

We included all variables that were significant predictors of subsequent myocardial infarction in the univariate analysis (table 1 ) in stepwise multivariate Cox proportional hazard models (table 3). Analysis excluding and including serum proinsulin at age 50 illustrates the influence of proinsulin on the predictive value of increase in blood glucose.

In the group being treated with antihypertensive drugs at age 60 the ratio of low density lipoprotein to high density lipoprotein cholesterol at age 50 and increase in fasting blood glucose were significant risk factors in the multivariate model. The impact of increase in blood glucose declined after addition of proinsulin to the models, but it was still a significant predictor of future myocardial infarction. In the group not receiving antihypertensive drugs the ratio of low density lipoprotein to high density lipoprotein cholesterol, serum proinsulin at age 50 , and increase in systolic blood pressure were independent predictors of subsequent myocardial infarction, but increase in fasting blood glucose was not a risk factor in this group.

We also performed the analysis in only the 977 participants for whom proinsulin concentrations were available. This reduction in sample size did not substantially change the results.

Serum proinsulin at age 50 was significantly correlated with increase in fasting blood glucose in the group with antihypertensive treatment $(r=0.32$, $\mathrm{P}<0.0001)$. A significant interaction also existed between proinsulin and antihypertensive treatment regarding increase in fasting blood glucose $(\mathrm{P}=0.0004)$. The men with the highest proinsulin concentrations at baseline showed the greatest increases in fasting blood glucose concentrations between the ages of 50 and 60 , especially in the group receiving antihypertensive treatment during this period (figure). A significant relation $(r=0.47, \mathrm{P}<0.0001)$ existed between the change in fasting blood glucose and the average of fasting blood glucose at age 50 and 60 , indicating that no regression towards the mean occurred.

\section{Discussion}

Our study shows that an increase in fasting blood glucose between the ages of 50 and 60 and high concentrations of circulating proinsulin are important risk factors for development of acute myocardial infarction after age 60 in men receiving antihypertensive treatment with $\beta$ blockers, thiazide diuretics, or both. In 
previous prospective cohort studies treatment with $\beta$ blockers or diuretics was associated with increased risk of diabetes, ${ }^{7810}$ probably owing to increased insulin resistance during treatment. ${ }^{56}$ However, in a more recent and larger study risk of diabetes was associated with $\beta$ blockers but not with diuretics. ${ }^{11}$ The doses of diuretics in that study were probably lower than in earlier investigations, which might explain why diuretics did not increase the risk of diabetes. ${ }^{18}$

The relation of insulin resistance to coronary heart disease has been well established, ${ }^{19}$ but the influence of the metabolic changes induced by antihypertensive treatment on the risk of myocardial infarction has been questioned. In contrast to our data, Samuelsson et al found that development of diabetes mellitus related to antihypertensive treatment did not increase the risk of coronary events. However, that study used diabetes mellitus (fasting glucose $>7.0 \mathrm{mmol} / \mathrm{l}$ ) as a categorical variable, and a categorical grouping based on fasting blood glucose diminishes the statistical capacity of detecting an effect. Furthermore, the confidence interval of the risk associated with drug related diabetes mellitus was wide. An effect of drug related diabetes mellitus on the risk of myocardial infarction should therefore not be excluded on the basis of the data from that study. ${ }^{15}$

In our study the incidence of myocardial infarction was significantly higher in the group with antihypertensive treatment than in participants without such treatment at age 60. A Swedish study in which hypertensive men had higher mortality from cardiovascular disease than non-hypertensive men presented similar findings. ${ }^{20}$ In our study an increase in fasting blood glucose, a high ratio of low density lipoprotein to high density lipoprotein cholesterol, and high serum proinsulin at baseline were significant risk factors for myocardial infarction after age 60 in the group with antihypertensive treatment. In spite of the treatment, the blood pressure at age 60 was not normalised, but neither baseline blood pressure nor achieved blood pressure at age 60 was related to the incidence of myocardial infarction.

In the multivariate analysis the impact of increase in fasting blood glucose on the risk of myocardial infarction was independent of baseline lipids, fasting insulin and glucose, body mass index, and blood pressure. However, when proinsulin was added to the

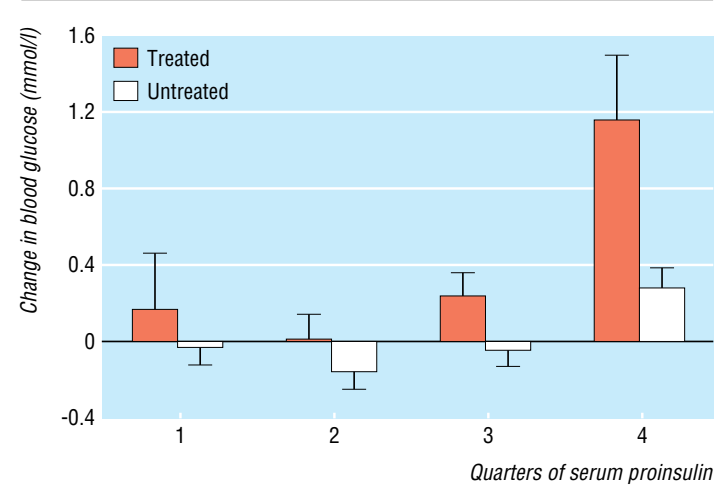

Mean (plus standard error of the mean) change in blood glucose between age 50 and 60 according to serum proinsulin levels at age 50 in the groups with and without antihypertensive treatment at age 60

\section{What is already known on this topic}

Patients with hypertension are resistant to insulin stimulated glucose uptake and are hyperinsulinaemic compared with normotensive controls

Treatment with $\beta$ blockers and thiazide diuretics further increases insulin resistance, thereby increasing the risk of developing type 2 diabetes mellitus or impaired glucose tolerance

The influence of metabolic changes induced by antihypertensive treatment on the risk of myocardial infarction has been questioned

\section{What this study adds}

Men who received antihypertensive treatment showed a larger increase in blood glucose during a 10 year period than those without such treatment

Increase in blood glucose during antihypertensive treatment was a significant, independent risk factor for myocardial infarction in men with an insulin resistant state at baseline

models the predictive power of increase in fasting blood glucose declined, indicating that insulin resistance at baseline, for which proinsulin may be a marker, ${ }^{21}{ }^{22}$ explains a part of the predictive power of the induced increase in fasting blood glucose in hypertensive patients. However, when the impact of insulin resistance (proinsulin concentration) was taken into account the deleterious effect of the increase in fasting glucose was still significant, indicating that both insulin resistance and the metabolic consequences of the antihypertensive treatment could be deleterious.

We were not able to measure change in insulin resistance and indices of the metabolic syndrome between age 50 and 60, except for measurements of glucose parameters. In this setting changes in fasting glucose reflect change in insulin resistance, and it is possible that several factors of the insulin resistance syndrome were affected accordingly.

Blood glucose may also directly affect the development of arteriosclerosis by impairing endothelial function. It is known that patients with type 2 diabetes mellitus have impaired endothelial function, ${ }^{23}{ }^{24}$ and increased concentrations of blood glucose have been shown to impair endothelial function in healthy people..$^{25} 26$

A limitation of our study is that we analysed proinsulin in only $55 \%$ of the sample. However, when we restricted the Cox proportional hazard analysis to participants with proinsulin determinations we obtained essentially the same, still significant, results, implying that no bias was introduced by the limited number of observations with proinsulin determinations.

Another limitation of this study is that the sample was restricted to 50 to 60 year old white men. In order to make these observations more generalisable, the findings must be replicated in other populations and in women.

In conclusion, we have shown the existence of an insulin resistant state with elevated proinsulin concen- 
trations resulting in increased fasting blood glucose concentrations during antihypertensive treatment with $\beta$ blockers and thiazide diuretics. Both the insulin resistant state and the induced metabolic disturbance were associated with increased risk of developing myocardial infarction.

Contributors: KD wrote the paper and did parts of the statistical analysis. HL is one of the founders of the Uppsala longitudinal study of adult men and was also involved in writing this paper. LL originated the idea and was involved in writing the paper and statistical analysis. BZ initiated the proinsulin analysis and helped with statistical analysis. LB did parts of the statistical analysis. Nicholas Hales, Cambridge, United Kingdom, analysed proinsulin. $\mathrm{KD}$ will act as guarantor.

Funding: Grants from Ernfors Fund for Diabetes Research, the Foundation for Geriatric Research, "Förenade Liv" Mutual Group Life Insurance Company, the Medical Faculty at Uppsala University, the Swedish Council for Planning and Coordination of Research (No. A19-5/62), the Swedish Diabetes Association, the Swedish Medical Research Council (No. 5446), the Swedish National Association Against Heart and Lung Disease, and Trygg Hansa Research Fund.

Competing interests: HL has acted as a consultant (currently for Solvay) for and has received funds for research from several companies that may either gain or lose financially from the results of the study. LL is partially employed as medical adviser at Astra Zeneca R\&D, Mölndal, Sweden.

Ethical approval: Local ethics committee of the medical faculty at Uppsala University, Uppsala, Sweden.

1 Ferrannini E, Buzzigoli G, Bonadonna R, Giorico MA, Oleggini M, Graziadei L, et al. Insulin resistance in essential hypertension. $N$ Engl J Med 1987;317:350-7.

2 Pollare T, Lithell H, Selinus I, Berne C. Application of prazosin is associated with an increase of insulin sensitivity in obese patients with hypertension. Diabetologia 1988;31:415-20.

3 Pollare T, Lithell H, Berne C. Insulin resistance is a characteristic feature of primary hypertension independent of obesity. Metabolism 1990;39:167-74.

4 Reaven GM. Relationship between insulin resistance and hypertension. Diabetes Care 1991;14(suppl 4):33-8.

5 Pollare T, Lithell H, Selinus I, Berne C. Sensitivity to insulin during treatment with atenolol and metoprolol: a randomised, double blind study of effects on carbohydrate and lipoprotein metabolism in hypertensive effects on carbohydrate and

6 Pollare T, Lithell H, Berne C. A comparison of the effects of hydrochloroPollare T, Lithell H, Berne C.A comparison of the effects of hydrochlorothiazide and captopril on glucose and lipid n
hypertension. N Engl J Med 1989;321:868-73.

7 Lundgren H, Bjorkman L, Keiding P, Lundmark S, Bengtsson C. Diabetes in patients with hypertension receiving pharmacological treatment. $B M$ 1988;297:1512.
8 Skarfors ET, Selinus KI, Lithell HO. Risk factors for developing non-insulin dependent diabetes: a 10 year follow up of men in Uppsala. BMJ 1991;303:755-60.

9 Sowers JR. Is hypertension an insulin-resistant state? Metabolic changes associated with hypertension and antihypertensive therapy. Am Heart J 1991;122:932-5.

10 Mykkanen L, Kuusisto J, Pyorala K, Laakso M, Haffner SM. Increased risk of non-insulin-dependent diabetes mellitus in elderly hypertensive subjects. J Hypertens 1994;12:1425-32.

11 Gress TW, Nieto FJ, Shahar E, Wofford MR, Brancati FL. Hypertension and antihypertensive therapy as risk factors for type 2 diabetes mellitus: and anthyperensive therapy as risk factors for type 2 diabetes mellitus:

2 Fuller JH, Shipley MJ, Rose G, Jarrett RJ, Keen H. Coronary-heart-disease risk and impaired glucose tolerance: the Whitehall study. Lancet risk and imp

13 Castelli WP. Epidemiology of coronary heart disease: the Framingham study. Am J Med 1984;76(2A):4-12.

14 Ramsay LE, Yeo WW, Jackson PR. Diabetes, impaired glucose tolerance and insulin resistance with diuretics. Eur Heart J 1992;13(suppl G):68-71.

15 Samuelsson O, Pennert K, Andersson O, Berglund G, Hedner T, Persson $\mathrm{B}$, et al. Diabetes mellitus and raised serum triglyceride concentration in Bete diaber treated hypertension-are they c

16 Hedstrand H. A study of middle-aged men with particular reference to risk factors for cardiovascular disease. Ups J Med Sci Suppl 1975;19:1-61.

17 Sobey WJ, Beer SF, Carrington CA, Clark PM, Frank BH, Gray IP, et al. Sensitive and specific two-site immunoradiometric assays for human insulin, proinsulin, 65-66 split and 32-33 split proinsulins. Biochem J 1989;260:535-41,

18 Harper R, Ennis CN, Sheridan B, Atkinson AB, Johnston GD, Bell PM. Effects of low dose versus conventional dose thiazide diuretic on insulin action in essential hypertension. BMJ 1994;309:226-30.

19 Haffner SM. Epidemiology of insulin resistance and its relation to coronary artery disease. Am J Cardiol 1999;84(1A):11J-14J.

20 Andersson OK, Almgren T, Persson B, Samuelsson O, Hedner T, Wilhelmsen L. Survival in treated hypertension: follow up study after two decades. BMJ 1998;317:167-71

21 Phillips DI, Clark PM, Hales CN, Osmond C. Understanding oral glucose tolerance: comparison of glucose or insulin measurements during the oral glucose tolerance test with specific measurements of insulin resistance and insulin secretion. Diabet Med 1994;11:286-92.

22 Mykkanen L, Zaccaro DJ, Hales CN, Festa A, Haffner SM. The relation of proinsulin and insulin to insulin sensitivity and acute insulin response in subjects with newly diagnosed type II diabetes: the insulin resistance subjects with newly diagnosed type II diabetes:
atherosclerosis study. Diabetologia 1999:42:1060-6.

23 Williams SB, Cusco JA, Roddy MA, Johnstone MT, Creager MA. Impaired nitric oxide-mediated vasodilation in patients with non-insulindependent diabetes mellitus. J Am Coll Cardiol 1996;27:567-74.

24 McVeigh GE, Brennan GM, Johnston GD, McDermott BJ, McGrath LT, Henry WR, et al. Impaired endothelium-dependent and independent vasodilation in patients with type 2 (non-insulin-dependent) diabetes mellitus. Diabetologia 1992;35:771-6.

25 Sarabi M, Millgard J, Lind L. Effects of age, gender and metabolic factors on endothelium-dependent vasodilation: a population-based study. $J$ on endothelium-dependent
Intern Med 1999;246:265-74.

26 Williams SB, Goldfine AB, Timimi FK, Ting HH, Roddy MA, Simonson DC, et al. Acute hyperglycemia attenuates endothelium-dependent vasodilation in humans in vivo. Circulation 1998;97:1695-701.

(Accepted 30 January 2002) 